\title{
Proptosis in a family with the p16 Leuc-to-Prol mutation in the PMP22 gene (CMT 1E)
}

\author{
Proptose em uma família com a mutacão p16 Leuc-to-Prol no gene PMP22 (CMT1E) \\ Leandro Calia ${ }^{1}$, Wilson Marques Jr. ${ }^{2}$, Silmara P. Gouvea², Charles Marques Lourenço², Acary S. B. de Oliveira ${ }^{1}$
}

${ }^{1}$ Departamento de Neurologia, Universidade Federal Paulista (UNIFESP), São Paulo SP, Brazil;

${ }^{2}$ Departamento de Neurociências e Ciências do Comportamento, Faculdade de Medicina de Ribeirão Preto, Universidade de São Paulo (USP), Ribeirão Preto SP, Brazil. Correspondence: Acary S. B. de Oliveira; Universidade Federal de São Paulo, Departamento de Neurologia/Neurocirurgia, Disciplina de Neurologia Clínica; Rua Botucatu 740; 04023-900 São Paulo SP - Brasil; E-mail: acary.nunesp@gmail.com

Support: This study was partially supported by CAPES, CNPq, FAPESP, FAEPA and INCT Translational Medicine.

Conflict of interest: There is no conflict of interest to declare.

Received 03 November 2012; Accepted 12 November 2012.

Charcot-Marie-Tooth disease (CMT) is the most common neuromuscular disorder. The autosomal dominant and demyelinating type (CMT1) associated with duplication of the PMP22 gene (CMT1A) is the most prevalent subtype around the world, including Brazil ${ }^{1}$, and is typically associated with mild or moderate neuropathy. Point mutations in the same gene (CMT1E) are extremely rare ${ }^{2}$ and typically result in severe demyelinating neuropathies, including Dejerine-Sottas syndrome and congenital hypomyelinating neuropathy3. Interestingly, CMT1 patients harboring the same mutation may develop different manifestations, even in identical twins.

Herein, we present Brazilian siblings harboring a previously described point mutation in the PMP22 gene that manifested as an unexpected clinical phenotype.

\section{CASE REPORT}

Both patients, one female and one male, were observed at the Neuromuscular Outpatient Clinics, UNIFESP, at the ages of 29 and 38 years old, respectively. Their family history indicated that the father had similar manifestations. They reported normal development up to the age of 12 and 11 years old, respectively, at which point they developed dropped feet and began to trip and fall. Thereafter, their disease slowly progressed. A physical examination identified severe distal weakness and atrophy in both the lower and upper limbs. The pain, tactile and vibration senses were severely decreased distally, and tendon jerks were absent. The peripheral nerves were hypertrophic, and both patients had pes cavus. The sister had claw hands, while the brother had a hand tremor. Interestingly, both patients had bilateral proptosis with preserved ocular movements. No malformation, including craniostenosis, was detected. Nerve conduction studies were performed, and no sensory or motor potential was recorded in the limbs; the blink reflex demonstrated $\mathrm{R} 1$ and $\mathrm{R} 2$ responses with very prolonged latencies. After obtaining informed consent, DNA analyses demonstrated that the PMP22 duplication was absent, but a point mutation was detected in both patients at position c.T47C, resulting in p16 Leuc-to-Prol in the protein.

\section{DISCUSSION}

The mutation found in these patients has been reported previously in a family with a disabling phenotype (DejerineSottas), including severe distal weakness, marked proximal paresis, severe kyphoscoliosis and foot and hand deformities ${ }^{4}$. The nerve conduction velocities were extremely low, and pathology was more severe than typically observed for CMT1A.

The siblings described in our report had a typical CMT1 phenotype. Additionally, both had bilateral proptosis whose investigation for the known causes ${ }^{5}$ resulted negative, raising the possibility that it is related to the harbored mutation.

Familiar bilateral proptosis is an extremely uncommon condition that typically appears in patients with hypertireoidism or cranial malformations. Rare reports of patients with proptosis associated with peripheral neuropathy have been described in the literature. However, they are typically part of a more complex neurological picture, such as that observed in mitochondrial disorders (e.g., due to OPA1 mutations) or genetic dysmorphic syndromes (e.g., Cardiofaciocutaneous syndrome).

To the best of our knowledge, proptosis has not been described in CMT1A or CMT1E, but variability in the CMT1 phenotype is a well-known phenomenon, even in identical twins. Unfortunately, the remaining members of this family were not available for examination. The function of PMP22 is still extremely unclear. Apparently, there is no significant function related to the eyes or orbita. If the proptosis observed in these patients is truly related to the detected mutation, PMP22 must have an unknown function. 


\section{References}

1. Marques W Jr, Freitas MR, Nascimento OJM, et al. 17p duplicated CharcotMarie-Tooth 1A:characteristics of a new population.J Neurol 2005;252:972-979.

2. Siskind CE, Shy ME. Genetics of neuropathies. Semin Neurol 2011;31:494-505.

3. Marques WJr., Neto JM, BarreiraAA. Dejerine-Sottas' neuropathy caused by the missense mutation PMP22 Ser72 Leu.Acta Neurol Scand 2004:110:196-199.
4

Valentijn LJ, Baas F, Wolterman RA, et al. Identical point mutations of PMP-22 I Trembler-J mouse and Charcot-Marie-Tooth disease type 1A. Nature Genetics 1992;2:288-291.

5. Aronson JK, Ramachandran M. The diagnosis of art: exophthalmos Gustave Doré's ogre. J R Soc Med 2006;99:421. 